New Zealand journal of industrial relations, 1983, 8, 57-61

\title{
Thoughts on industrial relations for the eighties
}

\author{
J.W. Rowe*
}

\section{What do the remainder of the eighties hold for industrial relations in New Zealand?}

It would be a brave person who tried to give a definitive answer to that question, as New Zealand is at an industrial relations crossroads at the present time. It is vital to the future that on both sides of the table we address ourselves to the issues we are facing and are likely to face for the remainder of the decade.

The only answer one could give with any confidence would be - "change -, technological, economic and social."

\section{Where are we at present?}

We are sitting in the middle of an international economic recession which, like it or not, has very serious implications for every one of us, employer or worker, in New Zealand. Perhaps we are fortunate that we have been able to cushion some of the worst effects of the recession by borrowing overseas to sustain our economy through this difficult period so far. On the other hand, we may have been too successful in this. We may have been cushioned too well, with the result that a lot of us have not yet woken up to the hard reality that things are not as they were and that we cannot go on the way we have been in the past. The buoyant days of the late sixties and early seventies are gone. We may in time get back to the standards we had then but it will not be overnight and it will not be without a lot of effort and some radical changes of attitude on the part of many people and groups.

Our present industrial relations structure, and many of the attitudes which have developed within it, came out of those better days. With a buoyant economy and full employment we became conditioned to a system which could sustain steady wage improvement, not only in direct monetary terms, but also in indirect forms such as additional time-off without loss of pay.

Over a period of years, we have become accustomed to a fairly rigid system of wage movement, with one or two major trade awards setting a figure at the start of each wage round and by and large all the rest following slavishly behind that same figure, regardless of the ability of a particular industry or undertaking, or indeed the national economy, to meet that figure. The only real areas of point-scoring available once the base figure is set are those of gaining some minimal additional percentage or in gaining some new condition of employment whether by way of a new allowance, or additional time-off for some purpose or other. These in turn, of course, merely become flow-on targets for the next wage round for those unions which have missed out in the current year.

Such a system can work satisfactorily only for a time. The equalising process involved means, naturally enough, that some gain more than is justified while others gain less. Over a period, however, pressure must build up. Those workers who feel that their employers 
could afford to pay more than the wage round figure will become increasingly dissatisfied with their situation, as will those employers who clearly can only afford to pay less and who eventually cease their operation as a result.

That is the position we have reached. As the economic situation has deteriorated over the years, a greater number of employers have found their wage costs increasing beyond their capacity to recover. Today, a considerable number of these have either shed or failed to replace labour, or have ceased operation entirely. If more are not to follow, wage increases in those areas must remain minimal until the position improves. Under the existing structure, however, this would require the wage-round figures to remain minimal, which from the worker's view would not be acceptable in many instances.

Clearly there are many factors involved, and a balance needs to be struck between them, but the present situation is that the wage round system of the last several years is at bursting point and its continued operation can only create increasing dissatisfaction amongst employers and workers alike.

\section{Where do we then go from here?}

First and foremost, our attitudes and thinking need to be revised. We have to recognise that we are living in a changed and rapidly changing world, and we need to become flexible enough to cope with that change. What operated satisfactorily for the last decade is not neccessarily satisfactory for today. What we determine as appropriate for today will not necessarily be appropriate for tomorrow.

We must have regard to the ability to pay of the nation, the industry, and the undertaking. That implies also recognising inability to pay. We have to acknowledge that the fortunes of any industry can change from one year to another, or even overnight, with consequent effects on the rewards of all in that industry. And when I say all, I mean all, employees as well as shareholders.

We have to recognise that both groups have rights. For too long we have had one group or the other failing to acknowledge the rights of, and their interdependence with, the other. This is of course a two-edged sword. Just as shareholders have a right to expect a financial return for their investment in the undertaking which provides the jobs for the employees involved, equally in this day and age they and management must realise that those same employees have an interest in the undertaking, and are entitled to know what is happening in the organisation and how it is fareing.

We have to recognise that no single group has a right to a monopoly in respect of any aspect of the operation of the economy, whether this relates to financial gain, information or representation of interests. We have to recognise that both sides of the table have a common interest in the ongoing viability of the organisation, whether it is the nation or a single company, that both have a contribution to make and both have a right to expect a reasonable share of the returns. But if this is to be the case, then both sides have to accept an equal responsibility to accept the bad with the good. There is a need for a much greater element of trust and openness between workers and management. There is nothing like a lack of understanding for creating permanent mistrust.

What issues then do we have to face up to if we are to achieve some of these ends?

Some will be tempted to say that they can never be achieved, and on past achievement this is not a surprising view. However, if New Zealand is to prosper in future, we have to adopt a positive viewpoint and work on the assumption that they can be achieved. It will not happen overnight, nor will it be easy.

The overriding issue we have to face up to for the eighties would appear to be a revision of our wage fixing system to provide something more appropriate to our circumstances. While this is the overriding issue, however, and requires immediate attention, it will be an on-going exercise the success of which will depend largely on other issues which must be approached at the same time.

Of these, the main one must be communication. At all levels. Management must develop a practice of communicating with its workers. Far too often situations occur where the 
first management hears of an issue is when the union knocks on the door. Without criticism of the union, this should simply not happen. When it does, it is generally management's "fault" through having failed to establish and encourage the use of a communication link which would permit the workers to convey the issue directly to management.

The essence of industrial relations is, after all, communication. Management needs to recognise this and be prepared constantly to talk to and listen to its work force. We have geared our society and educational system to produce enquiring minds. Having done that, we must learn to live with the resulting situation in which every decision of those in authority is subject to question. Change is invariably unsettling to those affected and tends to create resistance amongst those who cannot see the necessity for it. Management which cannot communicate to its staff the reasons for changes is in for a hard time in an era of constant change.

Communication is of course a two-way exercise. Management which communicates to its work force without obtaining input or feedback will run into as much trouble eventually as it would without any communication. Greater emphasis will have to be given during the eighties to developing systems that will allow the work force a say in matters affecting the organisation and their employment before the decision making process is finalised. This does not mean that management abrogates its decision making responsibility - few workers would in any case wish to be saddled with that - it does, however, entail an acknowledgement that the work force has an interest in matters affecting it and should have an opportunity to put its point of view before decisions are reached on such matters. Greater attention will have to be given to developing systems for employee participation.

Training is another issue which will require considerable attention during this decade, particularly in the area of retraining existing members of the labour force to cope with changing processes and skill requirements. The average New Zealand worker has a demonstrated ability to turn his or her hand to just about any activity, and the potential to cope with substantial changes in the working process or environment is enormous. To take advantage of this potential, however, may require that we develop a system of funding to provide a satisfactory level of income during retraining where continued employment with the same employer is not possible. This will of course raise questions as to who is to provide the necessary training and who is to determine what is required, and who is to meet the bill. Aside from the question of retraining existing members of the labour force, there is the matter of training future members. To cope with future change, our educational system needs to provide individuals with a sufficiently broad knowledge base to enable them to keep pace with such change, but at the same time the syllabus must be relevant to the needs of industry and commerce which will be providing the jobs for those coming out of the system. There will need to be a greater liaison between that system and those providing the jobs to ensure that the former is capable of instituting changes in training at a sufficiently early stage to provide the necessary capabilities and qualifications when they are required. This will of course impose on the employer groups a consequent obligation to determine as accurately as possible their requirements in advance, which will require considerable attention to refining forward planning and projection techniques. With rapid and substantial change, particularly in technology, this will become increasingly difficult but increasingly essential, and the decade will require increasing and continual attention to management training to enable management effectively to fulfil its role.

Turning back from these issues to the central issue of a revision of the wage fixing system, several items can be seen which will be issues in reaching any outcome on such a revision. The form which collective bargaining should take in the future, and the nature of rewards for employment, are just two.

Part of the problem with the present system, from an employers' point of view, is the lack of regard the system has to the particular circumstances of an individual employing undertaking. Clearly there are difficulties in negotiating a national code of conditions of employment which will cater for all the varying circumstances which will be encountered in different undertakings within different industries in different parts of the country. The situation is compounded when different national awards, negotiated for separate trades or 
occupations, and containing widely varying conditions of employment, have to be applied within a single undertaking to employees working virtually side by side. Within the existing system this has tended to create a rigid relativity structure for conditions of employment as well as pay rates and it is debatable whether this is in the interests of either workers or employers. The parties to the wage-fixing system will need to consider whether better alternatives exist for setting both wage rates and conditions of employment, other than by national trades awards.

In conjunction with an examination of the most appropriate form of collective bargaining, there would appear to be a need to consider the nature of rewards for employment. Over the years a fairly unwieldy system has been built up, providing a multitude of small payments for all sorts of conditions of work in addition to the basic wage rate. Many of these are of very limited application throughout the labour force and very often are of such limited amount that the cost of processing them alone exceeds the value paid to the recipient. Nonetheless, wages and salaries clerks throughout the country are required to be aware of the existence of such provisions and ensure that payment is made in appropriate circumstances, however infrequently this might occur. Often these are relevant only to particular industries yet because of a national award system they become incorporated as additional payments generally inappropriate to the bulk of employment covered by that award. Where the incidence of a particular condition of work in any undertaking or industry is such as to differentiate genuinely the work required of workers employed therein from the norm, it may be more appropriate to recognise such differences in the wage rate for the job rather than by way of separate payments. Removal of these would go a long way towards simplifying agreements. As each negotiating round passes under the present system, industrial awards and agreements become more complex and difficult to follow, which in turn leads to poorer understanding of their correct application with greater consequent potential for industrial relations problems.

We need also to give serious thought to the effects of non-monetary conditions of employment, usually in the form of paid time-off. We tend to forget that while these do not involve a direct financial benefit to the worker receiving them, they nonetheless represent an additional employment cost to the employer. Such additional costs, although less obvious than direct monetary increases, can have just as direct an implication for the viability of the undertaking, but under the present rigid relativities of the national award system they are imposed on operations which are unable to sustain them, ultimately leading to the likelihood of further loss of employment through the inability of the undertaking to continue its operation.

In any restructuring of the wage-fixing system we must surely give consideration to some means of relating the rewards of employment to the fortunes of the industry or undertaking. Having regard to the vital need for New Zealand to increase its productivity, there would seem to be logic in relating a substantial portion of remuneration to productivity by way of bonus systems. With the advent of Closer Economic Relations with Australia, and the difficult situation being faced by our exporting organisations in other world markets, it is more important than ever today that New Zealand production is competitive. Bonus systems of payment, combined with profit sharing schemes, where practicable, may well be one means of moving towards this, ensuring that reward is commensurate with effort and that all contributors towards the product receive a fair share of the returns. There is, however, no room for "phoney" productivity schemes which are no more than camouflaged wage increases.

Following on from the substantial levels of unemployment being experienced at the present time, security of employment will be an issue to which we will have to direct attention in the coming years. Contrary to suggestions that some employers might wish to see a permanent pool of unemployment in the hope of holding down wage rates and allowing greater selectivity in recruitment, the fact is that unemployment does not benefit any sector of the community. Stability in the labour force is just as important to the employers as to employees. There will obviously be situations in which undertakings which become no longer viable will be forced to cease operation with a consequent displacement 
of labour. We therefore need to look at ways of minimising the disruptive features of such occurrences with better forward planning and a set of guidelines for retraining and, where necessary, relocation of those affected. In conjuction with this, we should be able to eliminate or rationalise the "redundancy" concept which is such a plague at the present time.

Notwithstanding the present international economic situation, New Zealand has a marvellous potential to provide an excellent standard of living for all of us. However, to regain this will require that we work for it. We can not have our rewards "up front", we have to earn them first.

"Industrial Relations" is not some mystic term. Its basic meaning is simply the relationship between employer and employee. It can be good or bad. As in any relationship, its success depends on recognition and respect for the rights of the other party or parties. If we accept this and reshape our thinking and actions to make it work, it will work. 